JINR Preprint

E2-95-474

Dubna, November 1995

\title{
On the universality of the $x$ and $A$ dependence of the EMC effect and its relation to parton distributions in nuclei
}

\author{
G.I. Smirnov. \\ Joint Institute for Nuclear Research, 141980 Dubna, Russia. \\ Current address: CERN, PPE, 1211 Geneva, Switzerland \\ email: gsmirnov@cernvm.cern.ch
}

\begin{abstract}
It is shown that the latest results from the NMC (CERN) and E665 (Fermilab) groups on $F_{2}^{A}(x) / F_{2}^{D}(x)$ obtained in the shadowing region bring new evidence of the universal $A$ dependence of distortions made in a free-nucleon structure function by a nuclear medium. The observed universality implies that one can consider separately hard $(A \leq 4)$ and soft $(A>4)$ parton distribution distortions. Soft distortions, which result in differencies between the deep-inelastic scattering cross-sections for nuclei with masses $A_{1}, A_{2} \geq 4$, can be explained as a consequence of the nuclear density variation, independent of $x$ in the range $0.001 \leq x \leq$ 0.7 .

It is found that nuclear shadowing begins at $x_{\mathrm{I}}=0.0615 \pm 0.0024$, independent of $A$, which is consistent with models that allow for threeparton recombination processes.
\end{abstract}

Submitted to: Physics Letters B 
Particular interest in experimental and theoretical studies of modifications to the nucleon structure function $F_{2}\left(x, Q^{2}\right)$ in nuclei, triggered by the discovery of the EMC effect, is explained by the expectation of finding a common description for both the free-nucleon and nuclear structure functions in the framework of quantum chromodynamics.

The effects of the distortion of a free-nucleon structure by a nuclear medium are usually observed as a deviation from unity of the ratio $r^{A}(x) \equiv F_{2}^{A}(x) / F_{2}^{D}(x)$, where $F_{2}^{A}(x)$ and $F_{2}^{D}(x)$ are the structure functions per nucleon measured in a nucleus of mass $A$ and a deuteron, respectively.

Typical experimental errors in the measurements of the $r^{A}(x)$ are often of the same order or larger than the values of distortions. In such a case, the results of a comparison of distortions obtained at fixed $x$ in different nuclear targets suffer from large uncertainties. In particular, this applies to measurements with light nuclei, such as helium and lithium, which are very important for an understanding of the $A$ dependence of the distortions that, by definition of $r^{A}(x)$, should show up for $A \geq 3$. On the other hand, the conventional approach, which represents the $A$ dependence at fixed $x$ by

$$
r^{A}=C A^{\alpha(x)}
$$

does not exploit the conservation of total nucleon momentum carried by partons. To put it another way, the distortion of the nucleon structure function by the nuclear medium at some point $x$ is unjustly considered as independent of the distortion observed at the adjacent point $x+\Delta x$. This has motivated the alternative approach, suggested in Ref. [1], which determines the $A$ dependence of distortions after summing them up over an interval $\left(x_{1}, x_{2}\right)$.

The analysis of the data on deep-inelastic scattering (DIS) of muons and electrons off nuclear targets performed in Ref. [1] demonstrates that the $A$ dependence of distortion magnitudes obtained in each of three regions under study - namely shadowing, anti-shadowing and the EMC effect region follow the same functional form, being different in the normalizing factor only. This observation gives strong evidence for the universality of the $x$ and $A$ dependence of distortions in all nuclei with mass $A \geq 4$.

In this paper we present new evidence for such universality, found in the analysis of recent data collected from the DIS of muons on nuclei by the NMC (CERN) [2, 3] and E665 (Fermilab) [4] collaborations. This data brings to 14 the number of nuclei studied in the DIS of muons and electrons, which offers a good opportunity for studying the $A$ dependence of distortions in the structure function in nuclei from ${ }^{4} \mathrm{He}$ to ${ }^{207} \mathrm{~Pb}$.

Below, we consider structure function distortions as independent of the $Q^{2}$ 
at which $r^{A}(x)$ is measured. This is justified by conclusions about the $Q^{2}$ independence of $r^{A}$ in the range $0.2 \mathrm{GeV}^{2}<Q^{2}<200 \mathrm{GeV}^{2}$ (c.f. Refs. [2]- [6]).

In Ref. [1] it was found that the $x$ dependence of $r^{A}(x)$ can be factorized into three parts in the region $0.003<x<0.7$, in accordance with the differences in the $r^{A}(x)$ behaviour found in the three intervals of the considered range namely the (1) shadowing, (2) anti-shadowing and (3) EMC effect regions:

$$
r^{A}(x) \equiv F_{2}^{A}(x) / F_{2}^{D}(x)=x^{m_{1}}\left(1+m_{2}\right)\left(1-m_{3} x\right) .
$$

The parameters $m_{i}, i=1-3$, can be treated as the distortion magnitude of the nucleon structure function introduced for each interval. There are two physical reasons for parametrizing $r^{A}(x)$ in the form of Eq. (2). First, as was shown in Ref. [7], the nucleon structure function behaves as $F_{2}(x) \sim x^{-\lambda}$ in the range of small $x$, which is motivated by BFKL dynamics. Hence, combinations such as $F_{2}^{A}(x) / F_{2}^{D}(x)$ should obey a power law as well. Second, the parameters $m_{2}$ and $m_{3}$ enter Eq. (2) in a manner similar to the suggestion of Ref. [8], whereby local nuclear density is related to the deviation of $r^{A}(x)$ from unity in the range $x>0.3$.

As is shown in Ref. [1], the use of Eq. (2) is justified in the range $0.5<Q^{2}<$ $200 \mathrm{GeV}^{2}$. Nuclear shadowing is then described by one term only, since for $x \ll 1$, Eq. (2) reduces to the relation

$$
r^{A}(x)=C x^{\alpha} .
$$

A similar rate of increase in shadowing with a decrease in $x$ was expected at high $Q^{2}$, due to the gluon fusion considered in Refs. [9]. The results of Ref. [1] thus indicate that the gluon fusion mechanism persists for $Q^{2}$ as low as $\sim 0.5$ $\mathrm{GeV}^{2}$. The NMC and E665 data obtained in the range below $x=0.003$ do not deviate from Eq. (3) until $x=9 \cdot 10^{-4}, Q^{2} \approx 0.2 \mathrm{GeV}^{2}$. At the lower values of $x$, which correspond in the kinematics of NMC and E665 to lower $Q^{2}$, the data indicate (c.f. Refs. [3, 1, 10, 11] ) a smooth transition to the values of photoabsorption cross-section ratios, and thus cannot reflect the distortions of parton distributions by the nuclear medium. Therefore, we considered the data in the range $Q^{2}>0.3 \mathrm{GeV}^{2}$, which excludes the transition region $x<0.001$.

The parameters $m_{i}$ were determined by fitting $r^{A}(x)$, measured on seven nuclear targets - $\mathrm{He}$ [2, 5], Li [3], C [3, 5], $\mathrm{Ca}$ [2, 5], Xe [10], $\mathrm{Cu}$ [12] and $\mathrm{Pb}$ [4] — with Eq. (2). We used in the fit the total experimental error determined by adding statistical and systematic errors at each point in quadrature. For each of seven nuclei, good agreement $\left(\chi^{2} /\right.$ d.o.f. $\left.\leq 1\right)$ with Eq. (2) was found, thus proving that the characteristic pattern of the structure function modifications, well described for the helium nucleus by Eq. (2), remains unchanged for heavier nuclei. We consider this a manifestation of the universality of the $x$ 
dependence of the distortions of the free-nucleon structure function in a nuclear environment.

The results of the fit are shown in Fig. 1. The obtained parameters $m_{i}$, which represent the distortion magnitudes, increase from their minimum value $m_{i}(\mathrm{He})$ at $A=4$ to $m_{i}(A) \approx 3 m_{i}(\mathrm{He})$ for $A>40$, indicating that distortions in heavy nuclei are independent of the size of the nucleus. Previously, saturation of distortions was observed in the EMC effect region from the analysis of data available in the range $4 \leq A \leq 197$ [1].

The parameters $m_{i}$ vary similarly with $A$ in all three intervals in which the distortions were depicted. This similarity was first observed in Ref. [1] on a smaller sample of data. The points in Fig. 1 are approximated by the following equation:

$$
m_{i}(A)=N_{i}\left(1-\frac{A_{\mathrm{S}}}{A}\right) .
$$

This coincides, except for the normalization parameter $N_{i}$, with the factor $\delta(A)$ suggested in Ref. [13] for explaining the $A$ dependence of the EMC effect:

$$
\delta(A)=N\left(1-\frac{A_{\mathrm{S}}}{A}\right)=N\left(1-\frac{1}{A^{1 / 3}}-\frac{1.145}{A^{2 / 3}}+\frac{0.93}{A}+\frac{0.88}{A^{4 / 3}}-\frac{0.59}{A^{5 / 3}}\right),
$$

where the number of nucleons $A_{\mathrm{S}}$ at the nuclear surface was obtained using a Woods-Saxon potential with parameters taken from Ref. [14]:

$$
A_{\mathrm{S}}=4 \pi \rho_{0} \int_{r_{0}(A)}^{\infty} d r r^{2} \frac{1}{1+e^{\left[r-r_{0}(A)\right]} / a}
$$

where $\rho_{0}=0.17 \mathrm{fm}^{-3}$ is the central nuclear density, $r_{0}(A)=\left(1.12 A^{1 / 3}-\right.$ $\left.0.86 A^{-1 / 3}\right) \mathrm{fm}$ is a nuclear radius, and $a=0.54 \mathrm{fm}$ is the nuclear surface diffusion coefficient.

Thus the three lines in Fig. 1, a, b and c, differ only in the normalization factor $N_{i}$, which was found to be $N_{1}=0.130 \pm 0.004$ for the shadowing region, $N_{2}=0.456 \pm 0.017$ for the anti-shadowing region and $N_{3}=0.773 \pm 0.020$ for the EMC effect region. As shown in the comparison of $m_{i}$ with Eq. (4), the $A$ dependence of the distortion magnitudes is consistent with that defined by Eqs. (14) and (6). In other words, our results give evidence for a universal $A$ dependence of the distortion magnitudes $m_{i}$ of the nucleon structure function in all three regions. This universality can be expressed in terms of the relative distortions, measured in nuclei $A_{1}$ and $A_{2}$ with the following relation:

$$
\frac{m_{1}\left(A_{2}\right)}{m_{1}\left(A_{1}\right)}=\frac{m_{2}\left(A_{2}\right)}{m_{2}\left(A_{1}\right)}=\frac{m_{3}\left(A_{2}\right)}{m_{3}\left(A_{1}\right)} .
$$



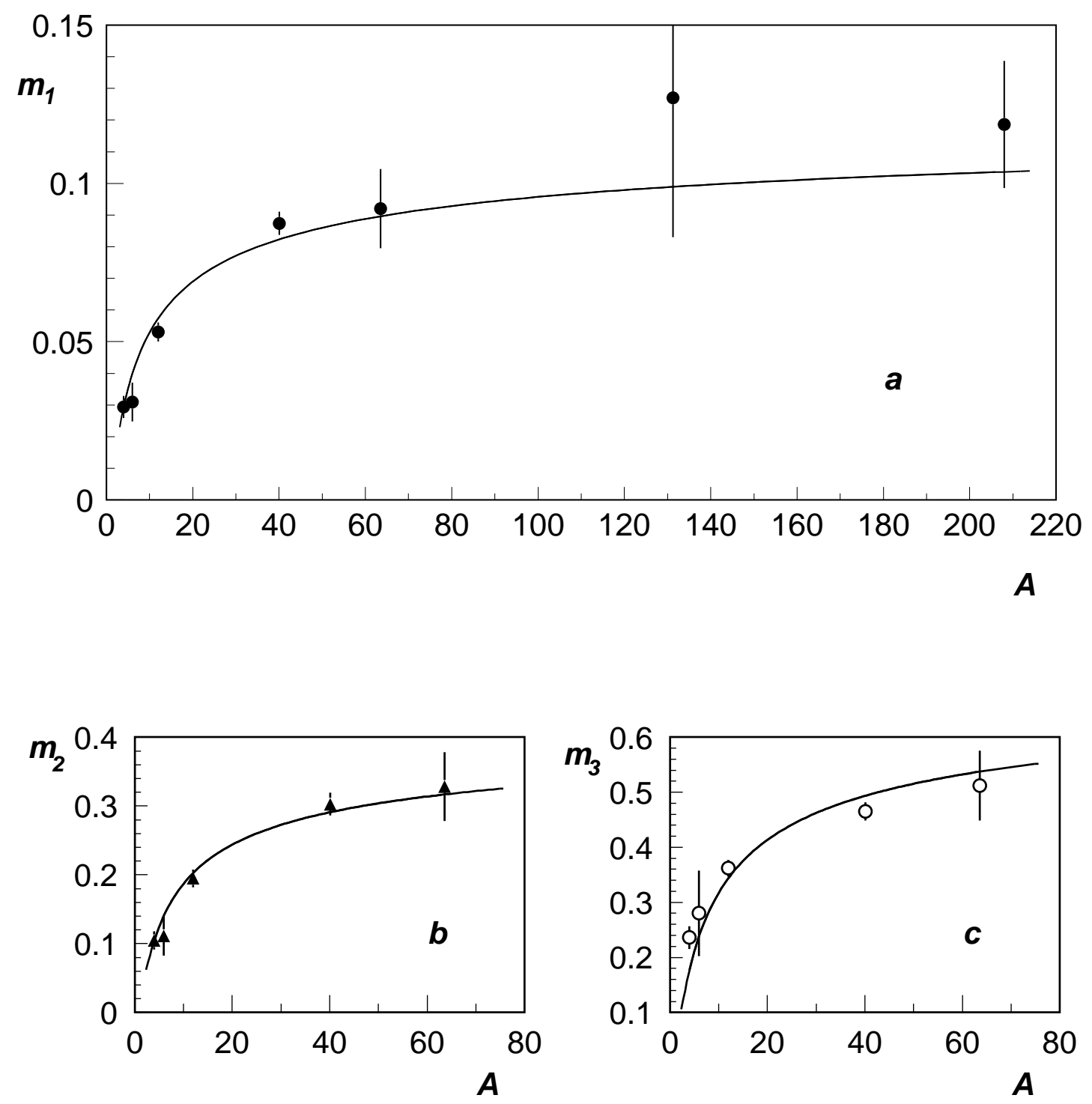

Figure 1: The parameters $m_{i}, i=1-3$, which define the magnitude of distortions of the nucleon structure function in a nuclear environment as a function of atomic mass $A$, determined in the regions of nuclear shadowing (a), antishadowing (b) and the EMC effect (c). Full lines show a variation in nuclear density given by the Woods-Saxon potential, with parameters fixed from the data on elastic electron-nucleus scattering. The three lines differ only in the normalization found from the fit to $m_{i}$. 
One can as well define the value of structure function distortion in units of that measured in the helium nucleus, $s_{\mathrm{h}}=m_{i}(A) / m_{i}(\mathrm{He})$. By definition, $s_{\mathrm{h}}=1$ for $A=4$, and, as follows from the obtained numerical values of $m_{i}, s_{\mathrm{h}}$ increases with $A$ to $\sim 3$ for heavy nuclei, independent of $x$.

The universality of the $x$ dependence of the nucleon structure function distortions implies that the positions of the three cross-over points $x_{i}, i=1-3$, in which $r^{A}(x)=1$, are $A$-independent if $A \geq 4$. Until recently, large experimental errors did not allow verification of theoretical predictions on the position and $A$ dependence of $x_{i}$, discussed in a number of publications (c.f. Refs. |15][18]). The situation has not improved for $x_{\mathrm{III}}(\sim 0.8)$, and one needs both higher statistical accuracy in $r^{A}(x)$ and a larger number of nuclei to establish whether $x_{\text {III }}$ is indeed $A$-independent. On the other hand, the data on $r^{A}(x)$ currently available in the EMC effect region made it possible to establish that, within experimental errors, the coordinate of the second cross-over point does not depend upon $A$ in the range $4 \leq A \leq 197$ and equals $x_{\mathrm{II}}=0.273 \pm 0.010$ [回].

We find $x_{\mathrm{I}}$ as an intersection point of a straight line $r^{A}(x)=1$, with $r^{A}(x)$ given by Eq. (3). The parameters $C$ and $\alpha$ have been found by fitting DIS data in the range $0.001<x<0.08$ on $\mathrm{He}, \mathrm{Li}, \mathrm{C}$ and $\mathrm{Ca}$ by $\mathrm{NMC}$ [2, 3], on $\mathrm{Cu}$ by EMC [12], and on $\mathrm{Xe} \mathrm{[10]} \mathrm{and} \mathrm{Pb}$ [4] by E665.

Agreement between the data obtained on the same nuclear target from two different experiments is an absolutely necessary condition for including data from the two experiments in a study of the $A$ dependence. As shown in Ref. [1], the data on $r^{\mathrm{C}}(x)$ and $r^{\mathrm{Ca}}(x)$ from NMC and E665 are not consistent with each other in the range $x<0.1$ and thus can not be combined for the analysis. As in the case of the $A$ dependence of $m_{i}$, one would expect that the $A$ dependence of $x_{\text {I }}$ shows itself in the range $A<40$. Consequently, in order to minimize systematic errors we have kept in the analysis the data on $\mathrm{C}$ and Ca nuclei from the NMC, which complement the data on He and Li from the same collaboration. At the same time we have included the data on the lead nucleus, collected by E665 only. For consistency's sake we use the $r^{\mathrm{Pb}}(x)$ from Ref. [4 which was obtained using the NMC procedure of radiative corrections.

The values $x_{\mathrm{I}}$ obtained as a function of $A$ are plotted in Fig. 2. Similar to the behaviour observed earlier for $x_{\mathrm{II}}$, within experimental errors the results are consistent with $x_{\mathrm{I}}=$ const $\left(\chi^{2} /\right.$ d.o.f. $\left.=6.1 / 7\right)$ and correspond to $x_{\mathrm{I}}=0.0615$ \pm 0.0024 .

The $A$-independence of $x_{\mathrm{I}}$ demonstrated by the present analysis provides a clue for better understanding of the shadowing mechanism. As follows from the results shown in Fig. 2, the idea of the $A$-dependent $x_{\mathrm{I}}$, widely exploited 


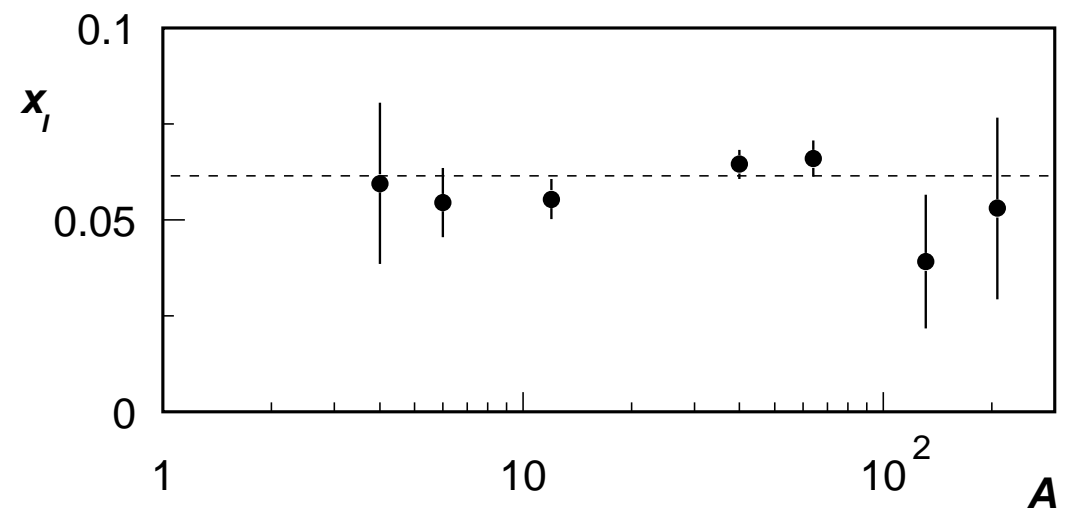

Figure 2: The coordinate of the first cross-over point $x_{\mathrm{I}}$ as a function of atomic mass $A$. The average value $\bar{x}_{\mathrm{I}}=0.0615$ is shown with a dashed line.

in models [16]-18], has to be discarded. Going back to the first discussion of the shadowing in the parton model [19], a number of papers relate the onset of the deviation of $r^{A}(x)$ from unity to the size of the region where the partons belonging to two or more neighbouring nucleons can be localized [16]-[18]. The position of $x_{\mathrm{I}}$ is related in Ref. [16] to the size of a nucleon, $R_{\mathrm{N}}$, and the number of overlapping nucleons $n$ :

$$
x_{\mathrm{I}}=\frac{1}{(n-1) 2 R_{\mathrm{N}} m_{\mathrm{N}}},
$$

where $m_{\mathrm{N}}$ is the nucleon mass. Taking our result for $x_{\mathrm{I}}$ and also the most precise value of the proton root-mean-square radius $<R_{\mathrm{E}}^{2}>_{p}^{1 / 2}=0.862 \pm 0.012$ $\mathrm{fm}$, obtained from the analysis of the data on elastic electron-proton scattering [20], we find that $n=2.98 \pm 0.08$.

The suggestion of ref. [13] to use the nuclear surface-to-volume ratio to explain the modification of the nucleon structure function in the EMC effect region has also been explicitly considered in Refs. [21, 22]. Our observation of the $A$ dependence of $m_{i}$ means that the nucleon structure is not modified if the nucleon belongs to the nuclear surface, not only in the EMC effect region, but also in the regions of nuclear shadowing and anti-shadowing. 
The parameters describing nuclear structure in Eq. (6) have been determined from the elastic scattering of electrons on nuclei (c.f. Ref. [23]). The same parameters allow one to reproduce with Eq. (国) the $A$ dependence of $m_{i}$, obtained from experiments with momentum transfers of three orders of magnitude higher. Thus, the results shown in Fig. 1 demonstrate remarkable consistency between experimental studies of nuclear structure from deep-inelastic and elastic scattering of leptons off nuclei.

The role of the nuclear surface-to-volume ratio in the observed modifications of the nucleon structure function can also be studied by means of the comparison of $F_{2}^{A_{1}}(x)$ and $F_{2}^{A_{2}}(x)$, when $A_{1}, A_{2} \geq 4$. The results of such measurements are expected soon from the NMC Collaboration [24]. When $F_{2}^{A}(x)$ is obtained in the DIS regime in the shadowing region, the ratio of the structure functions is described by Eq. (3), where the parameter $\alpha\left(A_{1} / A_{2}\right)$ is related to distortions $m_{1}(A)$ in a trivial way:

$$
\alpha\left(A_{1} / A_{2}\right)=m_{1}\left(A_{1}\right)-m_{1}\left(A_{2}\right) .
$$

Obviously, $\alpha\left(A_{1} / A_{2}\right)$ can also be calculated from the data on the EMC effect by using Eq. (田) and the normalization parameters $N_{i}$ found by our analysis. The similarity in the $A$ dependence of the $m_{i}$ justifies the use of Eq. (7) to relate $\alpha\left(A_{1} / A_{2}\right)$ to $m_{3}(A)$, even if deviations from Eq. (何) are found.

From the universality of the $x$ dependence of the distortions we expect that the coordinate of the first cross-over point determined from the ratios $F_{2}^{A_{1}}(x) / F_{2}^{A_{2}}(x)$ is $A$-independent and coincides with that determined by the present analysis.

Perturbative QCD provides a natural framework for the calculation of the modification to the structure function arising from the fusion of quarks, antiquarks and gluons [15, 25, 26]. As has been shown in Ref. [26] QCD (together with effects of $Q^{2}$ rescaling) is capable of describing the modifications to $F_{2}\left(x, Q^{2}\right)$ not only in the nuclear shadowing region, where it proved to be very successful, but also in the entire $x$ range. There remains, however, the problem of the role of two-, three- and four-parton fusion mechanisms in the QCD calculations. Judging from the agreement between the data on $r^{\mathrm{He}}(x)$ and calculations which assume either a two- [9] or three- [13] gluon fusion mechanism, one cannot give preference to either of the two approaches. New insight into this problem is provided by our results on $x_{\mathrm{I}}$, which should be considered as an argument in favour of contribution of the recombinations of gluons from three different nucleons.

Further improvement of the theoretical description of distortions in a freenucleon structure function is hardly possible until the mechanism responsible 
for the universality of the $x$ and $A$ dependence of the EMC effect is fully understood.

We suggest that modifications to the parton distributions of the nucleon bound in a nucleus evolve as a function of atomic mass $A$ in two stages. In the first stage, the distributions of partons belonging to the lightest nuclei, $2<A \leq$ 4 , are modified drastically compared to those of a free nucleon, thus distorting the structure function $F_{2}(x)$. These distortions, which can be observed in a ${ }^{4} \mathrm{He}$ nucleus as a characteristic oscillation of $r^{A}$ around the line $r^{A}=1$, remain frozen in shape in the second stage of distortions, which occur in nuclei with mass $A>4$. In contrast to the first stage, in the second there is no restructuring of parton distributions, which can change the shape of the oscillation described by Eq. (2). Instead, the distortions increase in magnitude throughout the entire $x$ range, following the functional form (四).

There are evidently two different mechanisms behind this picture, which we denote as hard or soft distortions, depending on whether $A \leq 4$ or $A>$ 4. Quantitatively, this can be expressed with the parameter $s_{\mathrm{h}}$, which rapidly changes in the range of hard distortions, from 0 to $1(\Delta A=2)$, and only slowly in the range of soft distortions, from 1 to $\sim 3(\Delta A \approx 200)$. A particular case of the hard distortion mechanism, which works at $A=4$, has been considered in Refs. [27, 28], in which EMC effect was explained by the 12-quark structure of nuclei.

In terms of the two-mechanism model, the experimental observations can be interpreted as follows: a) the positions of the three cross-over points are determined by hard distortions, and b) $x_{i}$ are $A$-independent in the range of soft distortions. In other words, hard distortions are saturated at $A=4$, which can be understood if modifications of parton distributions in the nuclear environment are closely related to short-range nuclear forces. In this picture $x_{\text {III }}$ should be different when it is obtained in ${ }^{3} \mathrm{He}$ and ${ }^{4} \mathrm{He}$ nuclei. Before such data are available one can not exclude the possibility that the saturation is reached at $A=3$.

In summary, we have shown that the recent data on the DIS of electrons and muons off nuclei bring new evidence for the universality of the $x$ and $A$ dependence of distortions of a free-nucleon structure function, $F_{2}(x)$, by a nuclear medium, when $A \geq 4$. Such universality and, in particular, the evidence for the $A$-independence of $x_{\mathrm{I}}$, imply that hard distortions of parton distributions are saturated at $A=4$ (or even at $A=3$ ) and that the observed differences between the DIS cross-sections for nuclei with masses $A_{1}, A_{2} \geq 4$ are due to soft distortions. The latter are similar in the shadowing, anti-shadowing and EMC effect regions, and vary from 1 in ${ }^{4} \mathrm{He}$ to $\sim 3$ in ${ }^{207} \mathrm{~Pb}$. They can be 
well understood as a nuclear density effect if the surface nucleons are excluded from consideration.

It has been found that nuclear shadowing begins at $x_{\mathrm{I}}=0.0615 \pm 0.0024$, which is consistent with models that relate $x_{\mathrm{I}}$ to a picture of the recombination of partons from three different nucleons.

The problem of describing modifications of $F_{2}(x)$ in a nuclear medium can thus be reduced to the derivation of $F_{2}^{\mathrm{He}}(x)$. We see further progress in this field in experimental studies of hard distortions of the structure function in the ${ }^{3} \mathrm{He}$ nucleus, and also in the search for possible deviations from the $A$ dependence of $r^{A}(x)$ defined by the surface-to-volume ratio (e.g. saturation of soft distortions in heavy nuclei).

\section{Acknowledgements}

The author would like to acknowledge helpful discussions with A.M. Baldin, S.B. Gerasimov, A.V. Efremov, L.L. Frankfurt, V.K. Luk'yanov, I.A. Savin and M.V. Tokarev. He is also grateful to E. Gabathuler, D. Kharzeev, B.Z. Kopeliovich, G. van Middelkoop, U.-G. Meissner, N.N. Nikolaev and V.D. Toneev for useful remarks.

\section{References}

[1] G.I. Smirnov, Yad. Fiz., 58, No. 9 (1995) 1712; Phys. At. Nucl. (Engl. Transl.), 58, No. 9 (1995) 1613; hep-ph-9502368.

[2] NMC, P. Amaudruz et al., Nucl. Phys. B 441 (1995) 3.

[3] NMC, M. Arneodo et al., Nucl. Phys. B 441 (1995) 12.

[4] E665, M.R. Adams et al., Z. Phys. C 67 (1995) 403; hep-ex-9505006.

[5] SLAC, J. Gomez et al., Phys. Rev. D 49 (1994) 4348.

[6] BCDMS, G. Bari et al., Phys. Lett. B 163 (1985) 282;

A.C.Benvenuti et al., Phys. Lett. B 189 (1987) 483.

[7] A.D. Martin, W.J. Stirling and R.G. Roberts, Phys. Rev. D 50 (1994) 6734.

[8] L. Frankfurt and M. Strikman, Nucl. Phys. B 250 (1985) 143;

L. Frankfurt and M. Strikman, Phys. Rep. 160 (1988) 235.

[9] V. Barone et al., Z. Phys. C 58 (1993) 541.

[10] E665, M.R. Adams et al., Phys. Rev. Lett. 68 (1992) 3266.

[11] NMC, A.Paić, Nuovo Cim., 107A (1994) 2141.

[12] EMC, J. Ashman et al., Z. Phys. C 57 (1993) 211.

[13] S. Barshay, Z. Phys. C 27 (1985) 443;

S. Barshay and D. Rein, Z. Phys. C 46 (1990) 215.

[14] A. Bohr and M. Mottelson, Nuclear Structure, W.A.Benjamin, Inc., New York, 1969. 
[15] J. Qiu, Phys. Rev. D 55 (1987) 555.

[16] E.L. Berger and J. Qiu, Phys. Lett. B 206 (1988) 141.

[17] F.E. Close and R.G. Roberts, Phys. Lett. B 213 (1988) 91.

[18] W. Zhu and J.C. Shen, Phys. Lett. B 235 (1990) 170.

[19] N.N. Nikolaev and V.I. Zakharov, Sov. J. Nucl. Phys. 21 (1975) 227; Phys. Lett. B 55 (1975) 397.

[20] G.G. Simon et al., Nucl. Phys. A 333 (1980) 381.

[21] S. Date et al., Phys. Rev. Lett. 52 (1984) 2344.

[22] I. Sick and D. Day, Phys. Lett. B 274 (1992) 16.

[23] R. Hofstadter, Ann. Rev. Nuclear Sci. 7 (1957) 231.

[24] NMC, M. Arneodo et al., To be submitted to Nucl. Phys. B.

[25] A.H. Mueller and J. Qiu, Nucl. Phys. B 268 (1986) 452.

[26] F.L. Close, J. Qiu, R.G. Roberts, Phys. Rev. D 40 (1989) 2820.

[27] H. Faissner and B.R. Kim, Phys. Lett. B 130 (1983) 321;

H. Faissner, B.R. Kim and H. Reithler, Phys. Rev. D 30 (1984) 900.

[28] L.A. Kondratyuk and M.Zh Shmatikov, Pis'ma Zh. Eksp. Teor. Fiz. 39 (1984) 324 (JETP Lett. 39 (1984) 389);

Yad. Fiz. 41 (1985) 498 (Sov. J. Nucl. Phys. 41 (1985) 317). 